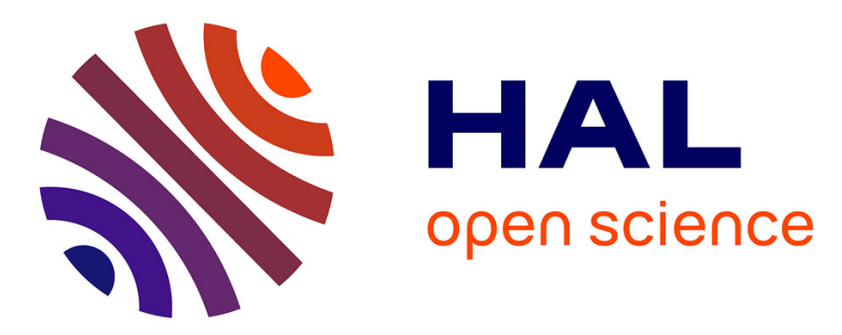

\title{
Refinement of orientation relations occurring in phase transformation based on considering only the orientations of the variants
}

\author{
Michel Humbert, Pierre Blaineau, Lionel Germain, Nathalie Gey
}

\section{To cite this version:}

Michel Humbert, Pierre Blaineau, Lionel Germain, Nathalie Gey. Refinement of orientation relations occurring in phase transformation based on considering only the orientations of the variants. Scripta Materialia, 2011, 64 (2), pp.114-117. 10.1016/j.scriptamat.2010.09.016 . hal-02401424

\section{HAL Id: hal-02401424 \\ https://hal.univ-lorraine.fr/hal-02401424}

Submitted on 10 Dec 2019

HAL is a multi-disciplinary open access archive for the deposit and dissemination of scientific research documents, whether they are published or not. The documents may come from teaching and research institutions in France or abroad, or from public or private research centers.
L'archive ouverte pluridisciplinaire HAL, est destinée au dépôt et à la diffusion de documents scientifiques de niveau recherche, publiés ou non, émanant des établissements d'enseignement et de recherche français ou étrangers, des laboratoires publics ou privés. 


\title{
Refinement of orientation relations occurring in phase transformation based on considering only the orientations of the variants
}

\author{
M. Humbert, ${ }^{*}$ P. Blaineau, L. Germain and N. Gey \\ Laboratoire d'Etude des Textures et Applications aux Matériaux, LETAM, CNRS FRE 3143 7078, ISGMP, Université de Metz, \\ F-57045 Metz Cedex 01, France
}

\begin{abstract}
The paper describes a general way to deduce the orientation relation (OR) occurring in phase transformation by considering only the orientations of a sufficient number of variants inherited from the same parent grain. Our current solution, which can be viewed as an optimization scheme using quaternion facilities, differs greatly from previous attempts proposed by other authors. The efficiency of the method is illustrated by searching for the OR determined from the variants of a transformed low-carbon steel.
\end{abstract}

Keywords: Phase transformations; Variants; Orientation relation determination; Steels

The mechanical properties of a material are strongly dependent on its microstructure, which derives mainly from phase transformation. At room temperature, the inherited phase is formed of variants linked to the parent high-temperature phase by orientation relations (ORs) (e.g. Nishiyama-Wassermann (NW) or Kurdjumov-Sachs (KS) ORs in steel transformation). Improving materials requires effective control of the orientation inheritance and of the variant selection. This implies an accurate knowledge of the parent and inherited microtextures as well as the OR. However, in general, the direct determination of the high-temperature parent microstructures is not easy. Several authors have therefore proposed methods to determine the parent microtexture when the ORs are known [1-3]. In this context, the determination of the representative ORs, which in steels may differ from those of NW or KS, is of particular interest. When a sufficient amount of the parent phase is retained at room temperature, it is possible to deduce a representative OR [4-9]. When this is not the case, Miyamoto et al. [10] briefly present a numerical method for determining the OR occurring in the steel phase transformation. This method uses the orientations of the variants to roughly evaluate the orientation of the parent

\footnotetext{
* Corresponding author. Tel.: +33 38731 5387; fax: +33 38731 5370; e-mail: michel.humbert@univ-metz.fr
}

austenite grain considering a defined OR (that of NW or of KS). The OR and the estimated parent orientation are then modified so that the sum of the deviations between the recalculated parent from each variant and their average becomes the smallest. When the minimum of the average deviation is reached, the OR and the parent orientation are assumed by the authors to be the best fitting.

We propose an analytical approach to determine the OR from a sufficient number of measured variants inherited from the same parent grain. The solution is quickly obtained analytically and can be adapted to any type of transformation.

Let us start with some brief reminders on the orientation links between parent and inherited orientations occurring in phase transformations. In a parent-todaughter phase transformation, the orientation of a given daughter variant can be characterized by a rotation $g_{v i}$ which is the product of several rotations (using the notations and conventions given in Ref. [11]):

$g_{v i}=D_{j} \cdot \Delta g \cdot P_{i} \cdot g_{0}$,

where $g_{0}$ is the rotation characterizing the parent crystal orientation. This orientation makes the sample reference frame parallel to that of the cubic parent. $P_{i}$ is one of the $n_{P}$ rotational symmetry elements of the parent phase. OR $\Delta g$, which describes the parent-to-daughter transformation, rotates the reference frame of the parent crystal relative to the reference frame of the daughter 
crystal. The choice of a rotation $P_{i}$ leads to a particular daughter variant. $D_{j}$ is one of the $n_{D}$ rotations of the rotational symmetry elements of the daughter lattice. It does not change the orientation of the daughter variant determined by $P_{i}$ and $\Delta g$. Analysis of relation (1) shows that the number of variants does not exceed $n_{P}$ and can be less ( 12 for NW and 24 for KS). Conversely, variant $g_{v i}$ can be related to a set of $n_{D}$ potential parent orientations according to the relation:

$P_{n} \cdot \Delta g^{-1} \cdot D_{m} \cdot g_{v i}$,

where $\Delta g^{-1}$ is the inverse OR and $P_{n}$ and $D_{m}$ are symmetry elements. Of course, the orientation $g_{0}$ is obtained from relation (2) when among the $n_{P}$ rotational symmetry elements of the parent phase, the symmetry element $P_{n}$ equals $P_{i}^{-1}$, and among the $n_{D}$ rotations of rotational symmetry elements of the daughter lattice, the symmetry element $D_{m}$ equals $D_{j}^{-1}$. The same type of relation is required irrespective of the variant considered. Consequently, the orientation $g_{0}$ corresponds to the unique common orientation shared by all the sets of potential parent (see Ref. [11]) and is expressed by:

$g_{0}=P_{n(i)} \cdot \Delta g^{-1} \cdot D_{m(j)} \cdot g_{v i}$,

where $P_{n(i)}$ and $D_{m(i)}$ are the specific known symmetry elements for each variant $i$ of the parent and inherited phases. To obtain a unique solution, the number of variants must be sufficient. By considering random choices of variants, one can easily show [1] that 4 different variants out of the 12 in the case of the NW OR always allow the parent orientation to be determined. This number equals 7 (out of 24) in the case of the KS OR. Of course some limited combinations of two specific variants also allow the unique solution to be found.

When the OR is not strict or when the orientations of the variants are determined with errors or are slightly modified by local plastic deformation induced by the transformation itself, relation (3) does not strictly hold. Nevertheless the rotations expressed by $P_{n(i)} \cdot \Delta g^{-1}$. $D_{m(j)} \cdot g_{v i}$ remain close to each other. Therefore in a first step, the parent orientation represented by $g_{0}$ and $P_{n(i)}, D_{m(j)}$ can be evaluated as in Ref. [11] for a given $\Delta g$ (NW or KS OR). When the differences between rotations $P_{n(i)} \cdot \Delta g^{-1} \cdot D_{m(j)} \cdot g_{v i}$ are not negligible, this indicates that the chosen OR is not the best representative of the real one. In that case, one can find a statistically more representative OR so that it reduces, on average, the difference between each rotation $P_{n(i)} \cdot \Delta g^{-1}$. $D_{m(j)} \cdot g_{v i}$. This implicitly leads also to obtaining the best estimate of the parent orientation $\left\langle g_{0}\right\rangle$ which is simply the mean value of the rotations $P_{n(i)} \cdot \Delta g^{-1} \cdot D_{m(j)} \cdot g_{v i}$ [11], once the best $\Delta g$ is found.

The search for this best representative OR can be seen as an optimization problem which can be efficiently solved by expressing rotations with quaternions. The product of rotations in relation (3) corresponds to a quaternion $\mathrm{Q}$ depending on the OR $\Delta g$, the other rotations being fixed and known (see above). For variant $\mathrm{i}$, the corresponding quaternion is $Q^{i}\left(\Delta g^{-1}\right)$. It can be seen that reducing the difference between the rotations $P_{n(i)} \cdot \Delta g^{-1} \cdot D_{m(j)} \cdot g_{v i}$ amounts to maximizing the norm of the sum of quaternions $Q^{i}\left(\Delta g^{-1}\right)$.

This is illustrated in Fig. 1. The norm of sum of two vectors (the quaternions considered here are unitary

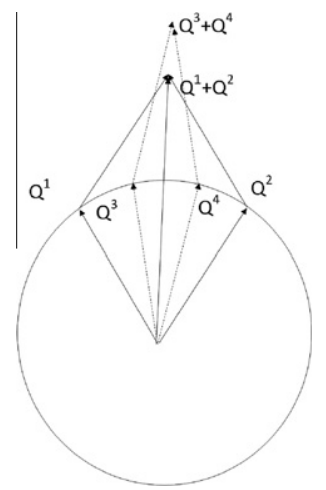

Figure 1. The norm of the sum of two unitary vectors is higher when the two vectors are closer.
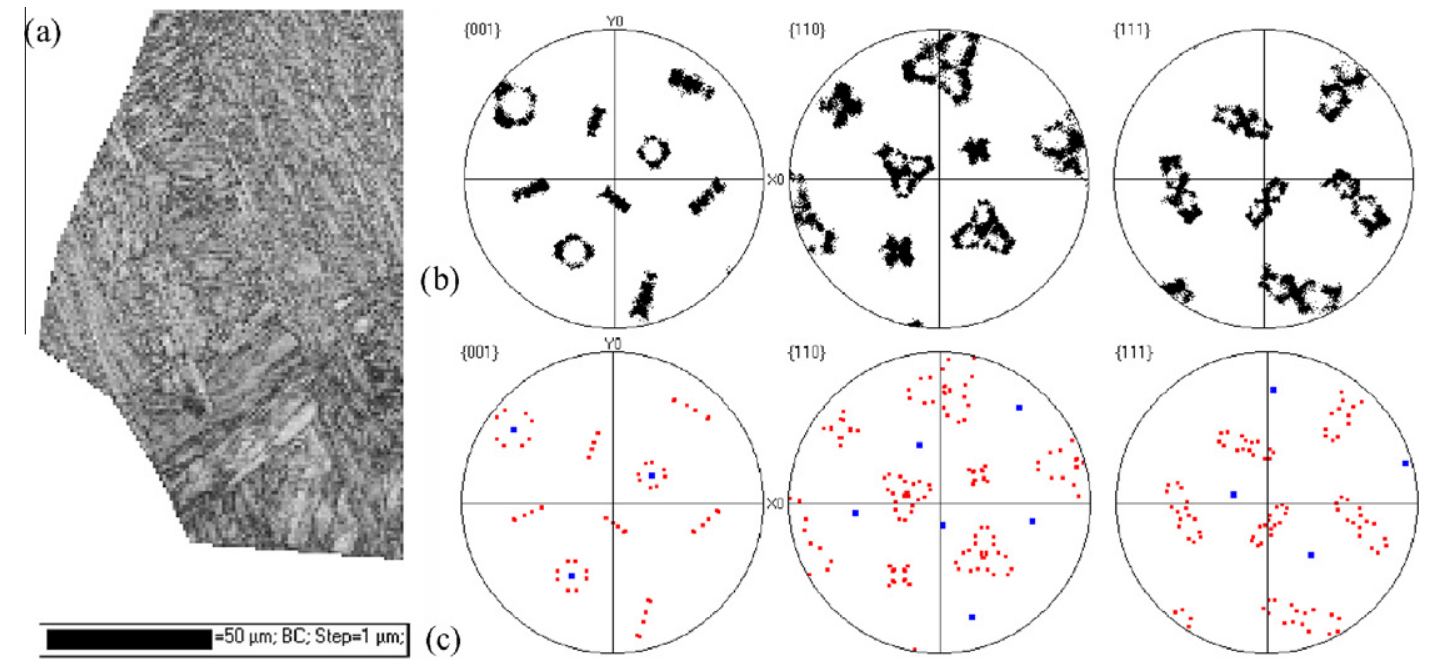

Figure 2. (a) microstructure of the investigated $\gamma$ transformed grain; (b) orientations of the $14,792 \alpha$ pixels represented by pole figures; (c) the same pole figures with the calculated parent $\gamma$ orientation (blue squares) and the recalculated variants (red squares) according to the OR determined. (For interpretation of the references in colour in this figure legend, the reader is referred to the web version of this article.) 
vectors of $R^{4}$ ) is higher when the two vectors become closer. At the same time their difference decreases. This result extents irrespective of the number of unitary vectors involved.

Using quaternion properties and multiplication rules [13], the components $\mathrm{zk}$ of a quaternion product $Z=P * Q \quad$ of quaternions $P \equiv\left(p_{0}, p_{1}, p_{2}, p_{3}\right) \quad$ and $Q \equiv\left(q_{0}, q_{1}, q_{2}, q_{3}\right)$ representing a product of rotations can be alternatively expressed by:

$$
\begin{aligned}
{\left[\begin{array}{l}
z_{0} \\
z_{1} \\
z_{2} \\
z_{3}
\end{array}\right]=} & {\left[\begin{array}{cccc}
p_{0} & -p_{1} & -p_{2} & -p_{3} \\
p_{1} & p_{0} & -p_{3} & p_{2} \\
p_{2} & p_{3} & p_{0} & -p_{1} \\
p_{3} & -p_{2} & p_{1} & p_{0}
\end{array}\right] \cdot\left[\begin{array}{l}
q_{0} \\
q_{1} \\
q_{2} \\
q_{3}
\end{array}\right] } \\
= & {\left[\begin{array}{cccc}
q_{0} & -q_{1} & -q_{2} & -q_{3} \\
q_{1} & q_{0} & q_{3} & -q_{2} \\
q_{2} & -q_{3} & q_{0} & q_{1} \\
q_{3} & q_{2} & -q_{1} & q_{0}
\end{array}\right] \cdot\left[\begin{array}{l}
p_{0} \\
p_{1} \\
p_{2} \\
p_{3}
\end{array}\right] }
\end{aligned}
$$

Thanks to these equivalent writings, each product of rotations such as $P_{n(i)} \cdot \Delta g^{-1} \cdot D_{m(j)} \cdot g_{v i}$ can be represented by a quaternion $Q^{i}$ whose components are related to the components of the quaternion $X \equiv\left(x_{0}, x_{1}, x_{2}, x_{3}\right)$ representing $\Delta g^{-1}$ by a relation of type:

$q_{k}^{i}=\sum_{l=1}^{4} M_{k l}^{i} \cdot x_{l}$

In this relation, $M_{k l}^{i}$ are the components of a $4 \times 4$ matrix $M$ which are functions of the components of quaternions describing $g_{v i}, P_{n(i)}$ and $D_{m(j)}$. An important point is that a rotation is represented by two opposite quaternions. One has to be aware of this when calculating the components of $M$.

The search for the most representative $\Delta g^{-1}$, expressed in the text above, amounts to maximizing the norm of the sum of quaternions $Q^{i}$ by finding the convenient unitary quaternion $X$.

For that purpose, one has to find the components of quaternion $X$ that maximize the expression:

$$
\left.\left.\left.\sum_{k=1}^{4} \sum_{l=1}^{4} \sum_{i} M_{k l}^{i}\right) \cdot x_{l}\right)^{2}+\lambda 1-\sum_{l=1}^{4}\left(x_{l}\right)^{2}\right) \text {. }
$$

This is a classical problem of maximization under constraints ( $X$ must be unitary) involving Lagrange multipliers $\lambda$. The solution is obtained by analysing the four eigenvalues $\lambda$ and the corresponding eigenvectors of the $4 \times 4$ matrix $A^{T} A$, the product of the transposed matrix $A$ and matrix $A$. The components of matrix $A$ are the sum of the corresponding components of matrix $M^{i}$ calculated from each variant:

$A_{k l}=\sum_{i} M_{k l}^{i}$.

The analysis of the four eigenvalues $\lambda$ of matrix $A^{T} A$ allows conclusions to be drawn on the multiplicity and relevance of the solution. When one eigenvalue is much greater than the three others, the maximization problem has a unique solution. In that case, the unitary eigenvector associated with this highest eigenvalue maximizes expression (6). It corresponds to the quaternion $X_{0}$ representing the best representative inverse OR $\left(\Delta g_{0}^{-1}\right)$. The multiple solutions arising when several eigenvalues have similar high values and their significances are not discussed in this paper.

Once the best representative solution $X_{0}$ is found, the value of the best estimated orientation $\left\langle g_{0}\right\rangle$ of the parent follows: it corresponds simply to the normalized sum of quaternions $Q^{i}\left(\Delta g_{0}^{-1}\right)$.

To illustrate the efficiency of this method, we have determined the OR and the parent orientation from the $\alpha$ (body-centred cubic) variants of a former $\gamma$ facecentred cubic grain of a low-carbon steel with a chemical composition (mass $\%$ ) of $0.2 \mathrm{C}, 2 \mathrm{Mn}, 20 \mathrm{ppm} \mathrm{P,} \mathrm{Fe} \mathrm{bal-}$ ance. The sample was heat-treated at $950{ }^{\circ} \mathrm{C}$ for $10 \mathrm{~min}$ followed by a water quenching plus a tempering at $300{ }^{\circ} \mathrm{C}$ for $5 \mathrm{~min}$. The orientation map was determined by electron backscatter diffraction with a step size of $1 \mu \mathrm{m} .14792$ pixels were measured on the grain being studied. The Kikuchi pattern quality map (Fig. 2a) gives an idea of its microstructure after transformation. $\left(\begin{array}{lll}1 & 0 & 0\end{array}\right)-\left(\begin{array}{lll}1 & 1 & 0\end{array}\right)-\left(\begin{array}{lll}1 & 1 & 1\end{array}\right)$ pole figures (Fig. 2b) express the orientations of the variants and show that the spread of the OR was not negligible.

In a first step, the parent orientation expressed by Euler angles (Bunge's definition [14]) $\left(125.26^{\circ}, 35.24^{\circ}\right.$, $\left.20.6^{\circ}\right)$ was evaluated using the $\mathrm{KS}$ OR $\left(\begin{array}{lll}1 & 1 & 1\end{array}\right) / / /$ $\left(\begin{array}{lll}1 & 1 & 0\end{array}\right)_{\alpha}$ and $\left[\begin{array}{lll}1 & -1 & 0\end{array}\right]_{\gamma} / /\left[\begin{array}{lll}1 & -1 & 1\end{array}\right]_{\alpha}$ corresponding to Euler angles $\left(5.77^{\circ}, 48.19^{\circ}, 5.77^{\circ}\right)$ ), according to the method described in Ref. [12]. The method proposed in the current paper was then applied to calculate the best representative OR and the best estimated parent orientation.

The highest eigenvalue of the product matrix $\left(A^{T} A\right)$ is very close to 1 , whereas the three others are very low. This indicates that the solution is unique. The corresponding eigenvector is equal to the quaternion representing $\Delta g_{0}^{-1}$ that maximizes the value of expression (6).

The OR $\Delta g$ calculated with this method is expressed by the following Euler angles $\Delta g \equiv\left(3.28^{\circ}, 45.09^{\circ}\right.$, $\left.6.23^{\circ}\right)$. This OR and the KS OR differ from $3.8^{\circ}\left(2.6^{\circ}\right.$ in the case of NW OR $\left(\left(\begin{array}{llll}1 & 1 & 1\end{array}\right)_{\gamma} / /\left(\begin{array}{lll}1 & 1 & 0\end{array}\right)_{\alpha}\right.$ and $\left[\begin{array}{lll}-2 & 1 & 1\end{array}\right]_{\gamma} / /\left[\begin{array}{lll}-1 & 1 & 0\end{array}\right]_{\alpha}$ corresponding to Euler angles $0^{\circ}$, $\left.\left.45^{\circ}, 9.73^{\circ}\right)\right)$. Consequently, on average, the $\left(\begin{array}{lll}1 & 1 & 1\end{array}\right)_{\gamma}$ plane is not exactly parallel to the $\left(\begin{array}{lll}1 & 1 & 0\end{array}\right)_{\alpha}$ plane $\left(1.38^{\circ}\right.$ away $)$, whereas the $\left[\begin{array}{lll}1 & -1 & 0\end{array}\right]_{\gamma}$ direction is $2.92^{\circ}$ away from the $\left[\begin{array}{lll}1 & -1 & 1\end{array}\right]_{\alpha}$ direction.

These values could suggest that the OR determined corresponds to that of GT (Greninger and Troiano $\left[\begin{array}{ll}15\end{array}\right) \quad\left(\left(\begin{array}{lll}1 & 1 & 1\end{array}\right)_{\gamma} / /\left(\begin{array}{lll}1 & 1 & 0\end{array}\right)_{\alpha}\left(1^{\circ}\right)\right.$ and $\left[\begin{array}{lll}1 & -1 & 0\end{array}\right]_{\gamma} / /\left[\begin{array}{lll}1 & -1 & 1\end{array}\right]_{\alpha}$ $\left(2.5^{\circ}\right)$ approximated by $(-1-1-1)_{\gamma} / /(-1-10)_{\alpha}$ and $\left[\begin{array}{lll}-17 & 12 & 5\end{array}\right]_{\gamma} / /\left[\begin{array}{lll}-17 & 17 & -7\end{array}\right]_{\alpha}$ according to Ref. [16] cited in Ref. [17]). Nevertheless the corresponding $\Delta g \equiv\left(3.21^{\circ}, 46.7^{\circ}, 7.5^{\circ}\right)$ differs from $\Delta g \equiv\left(3.28^{\circ}\right.$, $\left.45.09^{\circ}, 6.23^{\circ}\right)$ by $2^{\circ}$. At the same time, the calculated parent orientation equal to $\left(126.23^{\circ}, 34.96^{\circ}, 20.24^{\circ}\right)$, represented by blue squares on the pole figure in Fig. 2c, differs slightly from the first evaluation $\left(125.26^{\circ}, 35.24^{\circ}\right.$, $20.6^{\circ}$ ) by $0.8^{\circ}$. Fig. $2 \mathrm{c}$ shows also the good fitting of the 24 variants (red squares) calculated with the calculated representative OR and the experimental ones (Fig. 2b).

[1] P. Blaineau, L. Germain, M. Humbert, N. Gey, Solid 
[2] C. Cayron, J. Appl. Cryst. 35 (2007) 1183-1188.

[3] G. Miyamoto, N. Iwata, N. Takayama, T. Furuhara, ActaMat (2010), doi: 10.1016/j.actamat.2010.08.001.

[4] B. Gardiola, C. Esling, M. Humbert, K.E. Hensger, Adv. Eng. Mater. 5 (2003) 583-586.

[5] A. Lambert-Perlade, A.F. Gourgues, A. Pineau, Acta Mater. 52 (2004) 2337-2348.

[6] G. Nolze, Cryst. Res. Technol. 41 (2006) 72-77.

[7] G. Nolze, Cryst. Res. Technol. 43 (2008) 61-73.

[8] Y. He, S. Godet, P. Jacques, J.J. Jonas, Acta Mater. 54 (2006) 1323-1334.

[9] C. Cabus, H. Reglé, B. Bacroix, Mater. Charac. 58 (2007) 332-338.
[10] G. Miyamoto, N. Takayama, T. Furuhara, Scripta Mater. 60 (2009) 1113-1116.

[11] M. Humbert, H. Moustafhfid, M. Wagner, M.J. Philippe, Scripta Mater. 30 (1994) 571-576.

[12] M. Humbert, N. Gey, J. Appl. Cryst. 35 (2002) 401-405.

[13] B.K.P. Horn, J. Opt. Soc. Am. 4 (1987) 629-642.

[14] H.J. Bunge, Texture Analysis in Materials Science, Butterworths, London, 1982.

[15] A.B. Greninger, A.R. Troiano, J. Met. Trans. 185 (1949) 590-597.

[16] U. Dahmen, Acta Metall. 30 (1982) 63-73.

[17] Y. He, S. Godet, J. Jonas, J. Appl. Cryst. 39 (2006) 72-81. 